Brief Report:

\title{
Psychometric Properties of Short Version of Qualitative Checklist for Autism in Toddlers in a Sample of Iranian Toddlers: A Brief Report
}

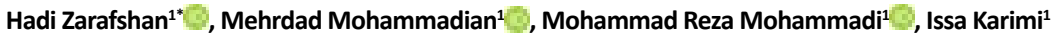

1. Department of Autism and Neurodevelopmental Disorders, Psychiatry and Psychology Research Center, Tehran University of Medical Sciences, Tehran, Iran.

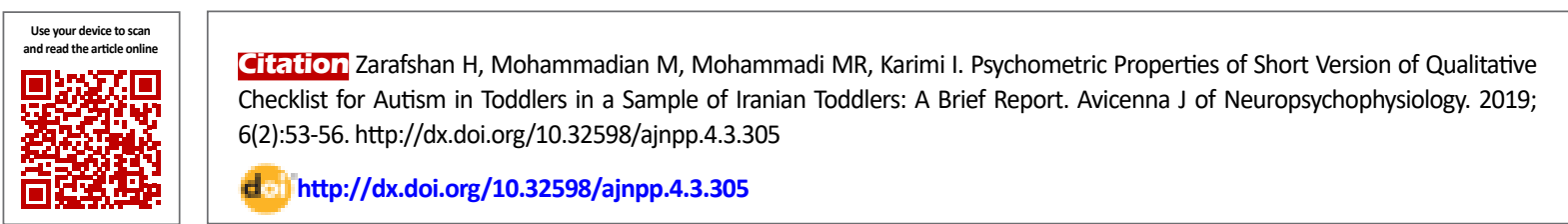

\section{(ㄷ) 0 (3)}

Article info:

Received: 02 Aug 2018

Accepted: 20 Jan 2019

Available Online: 01 May 2019

Keywords:

Autism spectrum disorder, Q-CHAT, Short version

\section{A B STRACT}

Background: Considering people with autism, early screening is necessary for early intervention. Parents report that questionnaires are effective tools for screening autism. The short version of the Checklist for Autism in Toddlers (Q-CHAT-10) is a new scale for screening autism in toddlers.

Objectives: The aim of this study was to evaluate the psychometric properties of the Persian version of Q-CHAT-10.

Materials and Methods: We analyzed the data of the full version of Q-CHAT. We only selected data regarding 10 items, which consisted of the short version of Q-CHAT.

Results: The typically developing group included 50 children with the mean age of 29.62 months, and the autism spectrum disorder group included 50 children with the mean age of 27.14 months. The mean total score was 2.1 and 6.46 for the typically developing group and the autism spectrum disorder group, respectively, which was significantly different $(t[98]=-11.52, P=0.000)$ The Cronbach's alpha coefficient of the checklist was 0.78 , and the test-retest reliability was 0.951 $(\mathrm{P}<0.001)$. The estimated area under curve was 0.935 .

Conclusion: The Persian version of Q-CHAT-10 has good reliability and predictive validity and it can be used for screening autism.

\section{* Corresponding Author:}

Hadi Zarafshan, PhD.

Address: Department of Autism and Neurodevelopmental Disorders, Psychiatry and Psychology Research Center, Tehran University of Medical Sciences, Tehran, Iran.

Tel: +98 (912) 7967889

E-mail: zarafshan84@gmail.com 


\section{Introduction}

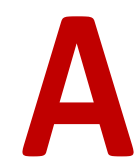

utism Spectrum Disorder (ASD) is a neurodevelopmental disorder characterized by social communication problems and restricted patterns of behaviors [1]. The prevalence of autism has increased in recent years by about $1 \%$ in The Diagnostic and Statistical Manual of Mental Disorders, Fifth Edition [2]. Early screening in toddlers for early intervention is highly recommended to decrease the negative effects of autism because, in the early course of development, the interaction between the plasticity of the brain and quality and intensity of interventions can lead to a better outcome in the future [3].

Using short parent-report questionnaires as the first step to screen individuals, who are at risk of autism, is common. These kinds of scales are reliable and do not need to specialist and a large amount of time to administer [4].

Checklist for Autism in Toddlers (CHAT) and modified CHAT are among screening tools, which have been used successfully in different countries to screen toddlers, who are at risk of autism [5]. Because of the changes in approach to autism as categorical diagnosis to dimensional diagnosis, the new version of this tool has been developed by their developers, which is called a Qualitative Checklist for Autism in Toddlers (Q-CHAT) [6]. It consists of 25 items answered by the caregivers of toddlers from 18-24 months on a 5 point Likert scale. Recently, it is increasingly used and its psychometric properties are evaluated in different languages and countries [7]. Since shorter questionnaires are more feasible, its short version was developed. The short version of Q-CHAT (QCHAT-10) consists of 10 items of its full version, which discriminates better between toddlers with autism and Typically Developing (TD) toddlers [8].

Previously, we have investigated the psychometric properties of the Persian version of Q-CHAT among Iranian toddlers [9]. Because of the shortness of appropriate and quick dimensional screening tools in the Persian language, in the present study, we aimed at investigating the psychometric properties of the short version of the Persian translation of Q-CHAT, using the data of our previous study [9].

\section{Materials and Methods}

We analyzed the data of the full version of Q-CHAT [9]. The original study was approved by the Ethics Committee of Iran University of Medical Sciences. As explained in full version report, in the first step, we prepared the Persian version of Q-CHAT. The items were translated to the Persian and, then, we checked the accuracy of the translation, using the back translation method. Next, we asked a few families to read and feedback us regarding the understandability of the items. When the final version of the questionnaire was prepared, we selected the appropriate sample and developed a checklist.

Based on the number of items of questionnaire and previous study, we considered a sample of 100 toddlers in our study. The sample included 50 TD toddlers and 50 toddlers with autism, who had been selected by convenience sampling method. The TD group was selected from a hospital nursery and based on their parents' reports; they had no history of developmental delay. To confirm that they do not have any developmental delay, they were evaluated by one of the authors.

The autistic group was selected among the patients of a psychiatric hospital and an autism center in Tehran, Iran. At first, children, who were suspected to have autism because of their developmental delay, were evaluated by a child psychiatrist based on The Diagnostic and Statistical Manual of Mental Disorders, Fourth Edition, Text Revision; those, who were diagnosed with autism, were referred to a second child psychiatrist to confirm their diagnosis. Children, who were diagnosed by both psychiatrists, were selected as the ASD group. Children with a severe physical disability or those, who were deaf or blind, were excluded from the study. We described the aim of the study and its procedure for parents and obtained the oral consonant form from them. After selecting the appropriate sample, the checklist was filled out by mothers of the selected children.

\section{Data analysis}

The data were analyzed by SPSS V. 19, using descriptive indices (frequency and Mean $\pm S D$ ) and inferential statistical tests (Independent t-test, Kolmogorov-Smirnov test, Cronbach's alpha, Pearson's r, and the receiver operating characteristic curve).

\section{Results}

The sample consisted of 50 toddlers with autism (ASD) and 50 TD toddlers. The Mean \pm SD age of the ASD group and the TD group was $27.14 \pm 7.68$ and $29.62 \pm 9.43$ months, respectively. There was no significant difference between the groups ( $\mathrm{t}[98])=-1.441, \mathrm{P}=0.153)$. In total, 38 males (76\%) and 12 females (24\%) were in the ASD group and 37 males (74\%) and 13 females (26\%) 


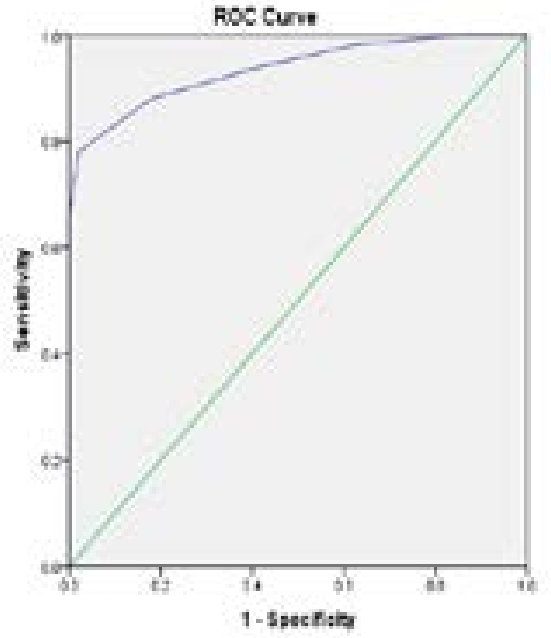

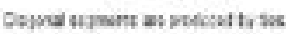

Figure 1. Area Under Curve (AUC)

AJNPP

were in the TD group. The Cronbach's alpha coefficient was $78 \%$ and the test-retest reliability was 0.951 $(P<0.01)$. The Mean \pm SD total score of $Q-C H A T-10$ of the ASD group and the TD group was $6.46 \pm 2.29$ and $2.1 \pm 1.37$, respectively. The mean score of the ASD group was significantly higher than that of the TD group $\left(t_{[98]}=-11.52, P<0.001\right)$.

The Area Under Curve (AUC) was 0.935 as an index of the overall predictive validity, indicating the ability of the Persian version of Q-CHAT-10 to discriminate between the ASD and TD groups (Figure 1).

\section{Discussion}

The Q-CHAT-10 can be used as a reliable tool to screen toddlers, who are at risk of autism. As it was expected, the mean scores of the ASD group were significantly more than that of the TD group ( $\left.\mathrm{t}_{[98]}=-11.52, \mathrm{P}<0.001\right)$. As there was no significant difference between the two groups based on the mean age and sex ratio, the observed differences in total scores can be reliable.

The Mean \pm SD of total score in the ASD group (6.46 \pm $2.29)$ is similar to Allison et al.'s study $(6.9 \pm 2.7)$, but in the TD group (2.1 \pm 1.37 ), it is slightly more than that of their findings (1.03 \pm 1.32$)$, which can be explained by differences between the age ranges of the TD groups in the two studies. The age range in Allison et al.'s study was 15-47 months, which is higher than that of our study. The Cronbach's alpha coefficient was $78 \%$ and the testretest reliability was $0.951(P<0.01)$, which indicates the reliability of scores. Table 1 Presents the sensitivity and specificity of different total scores of Q-CHAT-10.

The most important component of a good screening tool is its ability to discriminate individuals, who are at risk from a typical group. Estimating AUC can be an index of this ability. Based on the experts' opinion, in an ideal situation, in which a tool can discriminate all of the affected people from non-affected ones, the AUC will equal to 1 . As AUC is nearer to 1 , the screening tools are more useful [10]. The AUC in the present study is 0.935 , indicating the ability of the Persian version of $Q$ CHAT-10 in discriminating toddlers with ASD from the

Table 1. Sensitivity and specificity of different total scores of Q-CHAT-10

\begin{tabular}{|c|c|c|}
\hline Total Scores & Sensitivity & Specificity \\
\hline 1 & 0.01 & 0.14 \\
\hline 2 & 0.98 & 0.38 \\
\hline 3 & 0.94 & 0.58 \\
\hline 4 & 0.88 & 0.82 \\
\hline 5 & 0.78 & 0.99 \\
\hline 6 & 0.66 & 1 \\
\hline 7 & 0.54 & 1 \\
\hline 8 & 0.42 & 1 \\
\hline 9 & 0.22 & 1 \\
\hline 10 & 0.04 & 1 \\
\hline
\end{tabular}


TD group. This index in Allison et al.'s study was 0.965 , which is almost similar to that of our index.

It is recommended that the optimal cut-off score in a screening tool to identify affected individuals should have sensitivity between $70 \%-80 \%$ and specificity near to $80 \%$ [11]. In Allison et al.'s study, the recommended cut-off point is 3 . Based on the findings of this study, the cut-off point of 3 has a good sensitivity (93\%), but poor specificity (54\%). Instead, at the cut-off point of 4 , both sensitivity and specificity are at an acceptable level ( $88 \%$ and $82 \%$, respectively).

In sum, the Persian version of Q-CHAT-10 can be used as a reliable screening tool among Iranian toddlers. The cut-off point of 4 can be used as an optimal point for screening purposes.

\section{Ethical Considerations}

\section{Compliance with ethical guidelines}

All ethical principles were confirmed by Iran University of Medical Sciences.

Funding

This study used the data obtained from the previous study, which was financially supported by Iran University of Medical Sciences through a grant from the Mental Health Research Center.

\section{Authors' contributions}

All authors contributed equally in preparing all parts of the research.

\section{Conflict of interest}

The authors declared no conflict of interest.
[3] Dababnah S, Parish SL, Brown LT, Hooper SR. Early screening for autism spectrum disorders: A primer for social work practice. Children and Youth Services Review. 2011; 33(2):265-73. [DOI:10.1016/j. childyouth.2010.09.009]

[4] Barton ML, Dumont-Mathieu T, Fein D. Screening young children for autism spectrum disorders in primary practice. Journal of Autism and Developmental Disorders. 2012; 42(6):1165-74. [DOI:10.1007/ s10803-011-1343-5] [PMID]

[5] Yama B, Freeman T, Graves E, Yuan S, Campbell MK. Examination of the properties of the Modified Checklist for Autism in Toddlers ( $M$ CHAT) in a population sample. Journal of Autism and Developmental Disorders. 2012; 42(1):23-34. [DOI:10.1007/s10803-011-1211-3] [PMID]

[6] Allison C, Baron-Cohen S, Wheelwright S, Charman T, Richler J, Pasco G, et al. The Q-CHAT (Quantitative CHecklist for Autism in Toddlers): A normally distributed quantitative measure of autistic traits at 18-24 months of age: Preliminary report. Journal of Autism and Developmental Disorders. 2008; 38(8):1414-25. [DOI:10.1007/ s10803-007-0509-7] [PMID]

[7] Magiati I, Goh DA, Lim SJ, Gan DZQ, Leong J, Allison C, et al. The psychometric properties of the Quantitative-Checklist for Autism in Toddlers (Q-CHAT) as a measure of autistic traits in a community sample of Singaporean infants and toddlers. Molecular Autism. 2015; 6(1):40. [DOI:10.1186/s13229-015-0032-1] [PMID] [PMCID]

[8] Allison C, Auyeung B, Baron-Cohen S. Toward brief "red flags" for autism screening: the short autism spectrum quotient and the short quantitative checklist in 1,000 cases and 3,000 controls. Journal of the American Academy of Child \& Adolescent Psychiatry. 2012; 51(2):202-12. e7. [DOI:10.1016/j.jaac.2011.11.003] [PMID]

[9] Mohammadian M, Zarafshan H, Mohammadi MR, Karimi I. Evaluating reliability and predictive validity of the Persian Translation of Quantitative Checklist for Autism in Toddlers (Q-CHAT). Iranian Journal of Psychiatry. 2015; 10(1):64.

[10] Doi SA, Williams GM. Methods of clinical epidemiology. Vienna: Springer; 2013. [DOI:10.1007/978-3-642-37131-8]

[11] Glascoe FP. Screening for developmental and behaviora problems. Developmental Disabilities Research Reviews. 2005; 11(3):173-9. [DOI:10.1002/mrdd.20068] [PMID]

\section{References:}

[1] APA (American Psychiatric Association). Diagnostic and Statistical Manual of mental disorders (DSM- $\left.5^{\circ}\right)$. American Psychiatric Pub; 2013.

[2] Baio J. Prevalence of Autism Spectrum Disorders: Autism and developmental disabilities monitoring network, 14 Sites, United States, 2008. Morbidity and Mortality Weekly Report. Surveillance Summaries. Volume 61, Number 3. Centers for Disease Control and Prevention; 2012. 